SYNTHETIC BIOLOGY

\section{A platform for RNA virus cloning}

A study in Nature reports on the suitability of the yeast

Saccharomyces cerevisiae as a platform for the assembly and maintenance of diverse RNA virus genomes, including SARS-CoV-2, that enables their genetic manipulation and functional characterization.

During outbreaks, virus isolates are used for the development of diagnostics, in vivo models, antiviral therapeutics and vaccines. Viral genomes can be cloned from chemically synthesized DNA if the availability of virus isolates is limited, but established approaches using Escherichia coli are often inadequate to accommodate the large genomes of RNA viruses such as coronaviruses (family Coronaviridae).

Thao et al. applied transformation-associated recombination (TAR) cloning to the mouse hepatitis virus (MHV) containing a GFP gene, which has an established reverse-genetics platform. Overlapping DNA fragments covering the MHV-GFP genome and a TAR vector were transformed into yeast, upon which the DNA fragments assemble by homologous recombination, generating a yeast artificial chromosome (YAC) comprising the full-length viral cDNA. Notably, $>90 \%$ of screened clones showed correct assembly of the YAC, indicating high efficiency of assembly. Infectious virus was successfully recovered from two individual clones by isolating and linearizing the YACs for in vitro transcription to generate viral RNA, which was then transfected into the BHK-MHV-N hamster cell line, jointly with an mRNA encoding the MHV nucleocapsid protein, to produce and amplify the virus. Recovered virus exhibited the same replication kinetics as parental MHV-GFP.

The team set out to establish whether the synthetic genomics platform could be applied to MERS-CoV, using a low-copy bacterial artificial chromosome (BAC) to clone the virus from eight overlapping, PCR-amplified DNA fragments. The approach was also applied to a mutated MERS-CoV clone, which had a GFP gene inserted. YAC clone assemblies and virus rescues from cloned DNA were successful, ascertaining the applicability of this platform to a broader range of viruses, including genetically modified virus genomes.

Further experiments determined that viral genomes could be stably maintained and that this platform was applicable to other difficult to clone viruses, such as the ZIKA virus (family Flaviviridae) and human respiratory syncytial virus (family Paramyxoviridae), including directly from a clinical sample without prior knowledge of the virus genotype.

Strikingly, within 1 week of receiving synthetic DNA fragments of SARS-CoV-2 based on genome sequences released in January 2020, cloning and rescue of recombinant SARS-CoV-2 and SARS-CoV-2-GFP was achieved.

Taken together, this study showcases the utility of a synthetic genomics platform for the rapid generation and functional characterization of evolving RNA viruses during an outbreak from different starting materials, including virus isolates, cloned DNA, synthetic DNA or clinical samples.

Linda Koch

ORIGINAL ARTICLE Thao, T. T. N. et al. Rapid reconstruction of SARS-CoV-2 using a synthetic genomics platform. Nature https://doi.org/10.1038/s41586-020-2294-9 (2020)

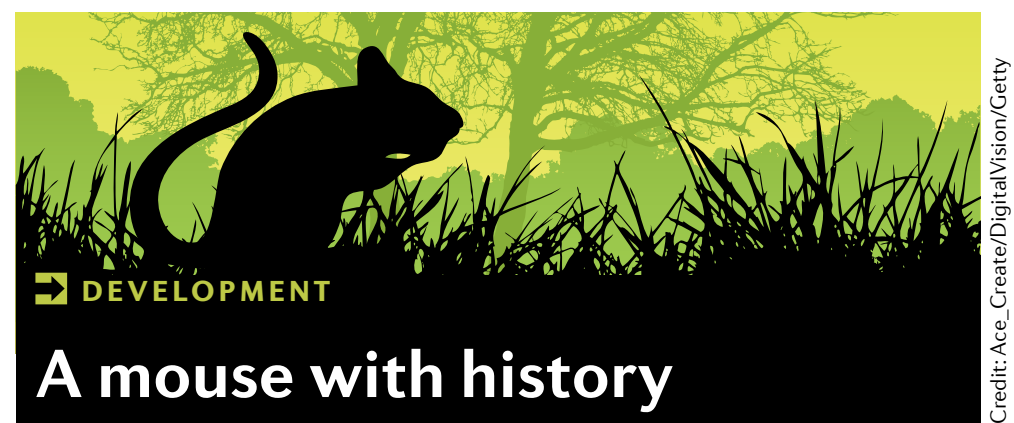

Engineered in vivo systems are emerging to characterize the lineage history and transcriptomic states of tissues in different organisms. However, existing CRISPR-based systems have typically required embryonic injection of editing reagents. A new study in Cell describes the CRISPR array repair lineage tracing (CARLIN) engineered mouse line that genomically encodes all the editing components, which can be activated in an inducible manner.

In their study, Bowling,

Sritharan et al. engineered a two-part locus into mice: an expression cassette for ten guide RNAs (gRNAs) was followed by a GFP gene in which a barcode array targeted by the gRNAs was inserted upstream of its polyadenylation site. As for several existing CRISPR-based lineage approaches, the rationale is that cutting and repairing the target barcodes leaves genetic 'scar' edits at these sites that are inherited by cellular descendants, and these scars can be diversified further by subsequent editing in derivative subclones. The placement of the target barcode array was chosen so that poly(A)-based RNA sequencing (RNA-seq) of edited single cells could reveal both their transcriptional state (based on the overall transcriptome) and their lineage history (based on sharing of edits in the expressed lineage barcode array).

To complete the set of required genomically encoded components, the authors crossed these mice to a line encoding the M2rtTA reverse tetracycline transactivator and TetO-regulated Cas9. This combination of elements in the resultant CARLIN mice allows Cas9 expression (and hence editing activity) to be induced in the presence of doxycycline.

The team carried out various proof-of-concept studies in the CARLIN mice and embryonic stem cells, such as optimizing doxycycline scheduling, adjusting single-cell RNA-seq protocols to capture both the lineage barcode and wider transcriptome with sufficient sensitivity, and demonstrating that CARLIN could recapitulate known lineage relationships in vivo.

The team particularly focused on the barcode diversity generated after doxycycline exposure, estimating that CARLIN can generate $\sim 44,000$ distinct edited barcode arrays. They generated a database of CARLIN-edited barcode frequencies and incorporated it into their analysis pipeline to help to distinguish informative lineage barcodes that are shared as a result of descent from a common cellular ancestor, versus less-informative barcodes that commonly arise independently in different clones.

As an example biological application, the authors showed that after haematopoietic depletion by 5 -fluorouracil, only a minor proportion of haematopoietic stem cells (HSCs) generated progeny that repopulate the haematopoietic system, and they identified transcriptional signatures that are associated with HSC repopulation potential.

The CARLIN mouse and its accompanying bioinformatic pipeline represent an important step towards opening up in vivo lineage tracing systems for wider adoption by the community. Its germline, inducible nature should allow it to address biological phenomena in diverse tissues in both young and adult mice.

Darren J. Burgess

ORIGINAL ARTICLE Bowling, S. et al. An engineered CRISPR-Cas9 mouse line for simultaneous readout of lineage histories and gene expression profiles in single cells. Cell https://doi.org/10.1016/j.cell.2020.04.048 (2020) RELATED ARTICLE Wagner, D. E. \& Klein, A. M. Lineage tracing meets single-cell omics: opportunities and challenges. Nat. Rev. Genet. https://doi.org/10.1038/s41576-020-0223-2 (2020) 